QUARTERLY OF APPLIED MATHEMATICS

VOLUME LXIX, NUMBER 2

JUNE 2011, PAGES 309-316

S 0033-569X(2011)01215-2

Article electronically published on March 9, 2011

\title{
REPRESENTATION OF DIVERGENCE-FREE VECTOR FIELDS
}

\author{
BY \\ CRISTIAN BARBAROSIE \\ CMAF, Av. Prof. Gama Pinto, 2, 1649-003 Lisboa, Portugal
}

\begin{abstract}
This paper focuses on a representation result for divergence-free vector fields. Known results are recalled, namely the representation of divergence-free vector fields as curls in two and three dimensions. The representation proposed in the present paper expresses the vector field as an exterior product of gradients and remains valid in arbitrary dimensions. Links to computer graphics and to partial differential equations are discussed.
\end{abstract}

1. Introduction. The aim of this paper is to study properties of vector fields having zero divergence, with particular emphasis on the representation of such vector fields in terms of a potential (which may be vector-valued). More precisely, a divergencefree vector field in $\mathbb{R}^{n}$ is expressed locally as an exterior product of $n-1$ gradients; see Theorem 5.3. This representation appears sometimes in textbooks on mechanics (especially fluid mechanics) and electromagnetism is a somewhat vague formulation; see the bibliographical comments at the end of Section 4. One motivation for seeking this type of representation result comes from computer graphics; see Section 3. Another motivation is related to elliptic partial differential equations; see Section 6,

The outline of the paper is as follows. Section 2 presents known representation results for curl-free vector fields and for divergence-free vector fields. In Section 3 some remarks are made on drawing two- and three-dimensional vector fields, with particular emphasis on divergence-free vector fields. Section 4 gives an intuitive description of the representation of a three-dimensional divergence-free vector field as the exterior product of two gradients; some bibliographical comments are included. In Section 5 the main representation result is stated and proven (Theorem [5.3). Section 6 discusses limitations of the results here presented and points out directions for future research.

Throughout this paper, $\Omega$ will be an open subset of $\mathbb{R}^{n}$. If $\varphi: \Omega \rightarrow \mathbb{R}$ is a differentiable function, we shall denote as usual by $\varphi_{, i}$ its partial derivative with respect to $x_{i}$. If $\vec{g}: \Omega \rightarrow \mathbb{R}^{n}$ is a vector field, we shall denote by $g_{i}$ its components; thus, $g_{i, j}$ will denote the derivative of the $i^{\text {th }}$ component of $\vec{g}$ with respect to $x_{j}$.

Received October 5, 2009

2000 Mathematics Subject Classification. Primary 26B12. Secondary 53A45, 53C65, 58A10, 58A25.

E-mail address: barbaros@ptmat.fc.ul.pt

(C)2011 Brown University 
2. Known representation results. Let $\vec{g}: \Omega \rightarrow \mathbb{R}^{n}$ be a $\mathcal{C}^{1}$ vector field. We say that $\vec{g}$ is curl-free if

$$
g_{i, j}=g_{j, i}, \forall i, j \in\{1,2, \ldots, n\} .
$$

REMARK 2.1. The above formula describes only the notion of a curl-free vector field, but does not define the curl of an arbitrary vector field. The curl of an arbitrary vector field $\vec{g}$ can be defined in several different ways. If $n=2$, curl $\vec{g}$ is the scalar function $g_{1,2}-g_{2,1}$ (the sign may vary from author to author). If $n=3$, curl $\vec{g}$ may be viewed as the vector field whose components are $\left(g_{1,2}-g_{2,1}, g_{2,3}-g_{3,2}, g_{3,1}-g_{1,3}\right)$ (again, signs may vary from author to author). A more sound definition, valid for arbitrary dimension, is to view curl $\vec{g}$ as the $n \times n$ anti-symmetric matrix of entries $g_{i, j}-g_{j, i}(1 \leq i, j \leq n)$. Whatever the formal definition chosen, one must have in mind that curl $\vec{g}$ has $n(n-1) / 2$ components.

It is well known that a curl-free vector field is locally a gradient. This representation can be extended to a global one if certain conditions hold on the behaviour of $\vec{g}$ at the boundary of $\Omega$. More precisely, the integral of the tangential component of $\vec{g}$ on each connected component of $\partial \Omega$ should vanish in order for a global potential $\varphi$ to exist (defined in the entire $\Omega$ ). These conditions are automatically verified if $\Omega$ is contractible. These conditions can be dropped if one allows $\varphi$ to be a multi-function.

Another direction for generalizing this representation result has to do with the regularity that we assume on $\vec{g}$. The regularity can be lowered, in which case the partial derivatives should be interpreted in the weak sense. If one takes $\vec{g} \in L^{2}(\Omega)$, the same local representation holds for a potential $\varphi \in H^{1}(V)$. Or, for $\vec{g} \in H^{-1}(\Omega)$, a potential is shown to exist in $L^{2}(V)$. See [3], section 2-6 and Corollary 2 in section 7-7.

We now focus on the notion of divergence. The divergence of an $n$-dimensional vector field $\vec{\jmath}: \Omega \subset \mathbb{R}^{n} \rightarrow \mathbb{R}^{n}$ is defined as $\operatorname{div} \vec{\jmath}=\jmath_{1,1}+\jmath_{2,2}+\cdots+\jmath_{n, n}$.

If $n=2$, there is a simple mapping between the operators curl and divergence. Let us define the matrix

$$
R=\left[\begin{array}{cc}
0 & -1 \\
1 & 0
\end{array}\right]
$$

representing a $90^{\circ}$ rotation in the plane. If $\vec{\jmath}$ and $\vec{g}$ are two-dimensional vector fields, then

$$
\operatorname{div} R \vec{g}=\operatorname{curl} \vec{g}, \operatorname{curl} R \vec{\jmath}=\operatorname{div} \vec{\jmath} .
$$

See [11, section 15.3, for an intuitive presentation of the effect of a $90^{\circ}$ rotation on a two-dimensional field.

Thus, in two dimensions, any result on curl-free vector fields applies to divergence-free vector fields by means of a $90^{\circ}$ rotation, the converse also being true. In particular, any divergence-free vector field is locally of the form $R \nabla \varphi$.

In higher dimensions there is no correspondence between the curl and the divergence of a vector field. As explained in Remark 2.1. the curl of a vector field consists of $n(n-1) / 2$ scalar functions, while the divergence is only one scalar function. Thus, when one specifies that a certain vector field has zero curl, much more information is provided than for zero divergence.

Very little is known about divergence-free vector fields in dimension $n \geq 4$. Some results are known in the literature for three-dimensional divergence-free vector fields. 
Theorem 2.2 (9], Theorem 3.2). Let $\Omega \subset \mathbb{R}^{3}$ be a bounded open set satisfying the Lipschitz condition. Let $\vec{\jmath}: \Omega \rightarrow \mathbb{R}^{3}$ be a vector field whose components belong to $L^{2}(\Omega)$ such that $\operatorname{div} \vec{\jmath}=0$ in $\Omega$ and

$$
\int_{\Gamma_{i}}\langle\vec{\jmath}, \vec{n}\rangle=0, i=1,2, \ldots, m
$$

where the integral should be understood in the sense of traces. $\Gamma_{1}, \Gamma_{2}, \ldots, \Gamma_{m}$ are the boundaries of the $m$ holes of $\Omega$, and $\vec{n}$ is the unitary vector normal to $\Gamma_{i}$. Then, there is a vector field $\vec{\varphi} \in H^{1}\left(\Omega ; \mathbb{R}^{3}\right)$ such that $\vec{\jmath}=\operatorname{curl} \vec{\varphi}$, that is,

$$
\begin{aligned}
& \jmath_{1}=\varphi_{2,3}-\varphi_{3,2}, \\
& \jmath_{2}=\varphi_{3,1}-\varphi_{1,3}, \\
& \jmath_{3}=\varphi_{1,2}-\varphi_{2,1} .
\end{aligned}
$$

It seems impossible to generalize the representation $\vec{\jmath}=\operatorname{curl} \vec{\varphi}$ for dimensions higher than three. This is one motivation for looking for different representations of divergencefree vector fields. Another motivation is presented in the next section.

3. Drawing vector fields. This topic (computer graphics) may seem unrelated to the subject of the present paper. However, when trying to draw vector fields nicely (that is, in a mathematically meaningful manner), one gets a feeling about their structure.

Drawing a vector field is a challenging task for those who do not content themselves with a fuzzy collection of arrows. The best way to represent a vector field is by drawing its trajectories, alone or tagged with tangent arrows. But choosing the right distance between trajectory lines is not easy. One often gets zones with a high density of lines, and other zones too empty. When the vector field has zero divergence, there is a physically sound way of representing the magnitude of the vectors with the aid of the distance between trajectories. Zones with high intensity correspond to small interline distance, while zones where the vector field has small magnitude correspond to a large distance between trajectories.

In two dimensions, this effect is easy to achieve if we remember that the vector field $\vec{\jmath}$ is the rotated gradient of a scalar potential $\varphi$. After computing $\varphi$, it suffices to draw its level lines (for equally spaced level values). These lines are trajectories of $\vec{\jmath}$, and there is an obvious relation between the interline distance and the magnitude of the gradient of $\varphi$, which is equal to the magnitude of $\vec{\jmath}$.

Can we generalize the above procedure for a three-dimensional vector field having zero divergence? The physical interpretation linking the intensity of the field to the density of drawn trajectories still holds. But the representation $\vec{\jmath}=\operatorname{curl} \vec{\varphi}$ does not help to choose and draw trajectory lines. We need a different type of representation. We want a function $\gamma$ whose level lines are trajectories of $\vec{\jmath}$. This means that the "potential" $\gamma$ should take values in $\mathbb{R}^{2}$. Thus, the level sets of each component of $\gamma$ would be surfaces, and intersections of these surfaces would be trajectories of $\vec{\jmath}$.

Although there is no point in drawing vector fields in dimension $n>3$, the procedure would be the same: a "potential" $\gamma$ should be constructed (taking values in $\mathbb{R}^{n-1}$ ), points should be chosen in $\gamma(\Omega)$, and the corresponding level lines should be drawn. 
As a side note, we mention that arbitrary vector fields (having divergence not necessarily zero) can be drawn using a similar technique. In zones where the divergence is positive, additional flow lines should start in order to increase the line density (thus decreasing the interline distance). In zones where the divergence is negative, some flow lines should be ended in order to decrease the line density. One systematic way to do this is to approximate the divergence of the vector field by a linear combination of Dirac masses, with weights of equal absolute value. At each positive Dirac mass, a new flow line should be started; near each negative Dirac mass, a flow line should be ended.

4. Divergence-free vector fields in three dimensions. The above considerations lead to the following idea: given a three-dimensional vector field $\vec{\jmath}$ with zero divergence, we are looking for two scalar functions $\gamma_{1}$ and $\gamma_{2}$ such that, at each point $x \in \Omega$, the vector $\vec{\jmath}(x)$ is tangent to the corresponding level surfaces. This means that the gradients of $\gamma_{1}$ and of $\gamma_{2}$ at $x$ should be orthogonal to $\vec{\jmath}(x)$.

As a consequence of the above considerations, it is natural to look for $\gamma_{1}$ and $\gamma_{2}$ such that

$$
\vec{\jmath}(x)=\nabla \gamma_{1}(x) \wedge \nabla \gamma_{2}(x), \forall x \in \Omega,
$$

where $\wedge$ denotes the exterior product between two vectors in $\mathbb{R}^{3}$ :

$$
\vec{a} \wedge \vec{b}=\left[\begin{array}{l}
a_{2} b_{3}-a_{3} b_{2} \\
a_{3} b_{1}-a_{1} b_{3} \\
a_{1} b_{2}-a_{2} b_{1}
\end{array}\right] .
$$

Before discussing the existence or construction of the potential $\gamma: \Omega \rightarrow \mathbb{R}^{2}$, we shall make various remarks.

Note that it is a trivial matter to check that, given any two functions $\gamma_{1}$ and $\gamma_{2}$ in $\mathcal{C}^{1}(\Omega)$, the vector field $\nabla \gamma_{1} \wedge \nabla \gamma_{2}$ has indeed zero divergence:

$$
\begin{aligned}
\operatorname{div}\left(\nabla \gamma_{1} \wedge \nabla \gamma_{2}\right)=\left(\gamma_{1,2} \gamma_{2,3}-\gamma_{1,3} \gamma_{2,2}\right)_{, 1} & +\left(\gamma_{1,3} \gamma_{2,1}-\gamma_{1,1} \gamma_{2,3}\right)_{, 2} \\
& +\left(\gamma_{1,1} \gamma_{2,2}-\gamma_{1,2} \gamma_{2,1}\right)_{, 3}=0 .
\end{aligned}
$$

Taking into account the algebraic definition of the exterior product of $n-1$ vectors in $\mathbb{R}^{n}$, one can see that the representation $\vec{\jmath}=\nabla \gamma_{1} \wedge \nabla \gamma_{2}$ of three-dimensional divergencefree vector fields is a natural generalization of $\vec{\jmath}=R \nabla \varphi$ for two-dimensional divergencefree vector fields.

Note also that the quantity $\nabla \gamma_{1} \wedge \nabla \gamma_{2}$ depends on $\gamma_{1}$ and $\gamma_{2}$ in a nonlinear manner (unlike previous representations; for instance, curl $\vec{\varphi}$ depends linearly on $\vec{\varphi}$ ). This nonlinear character brings new difficulties, which show up when one tries to lower the regularity of the functions (suppose $\gamma_{1}, \gamma_{2} \in H^{1}$; then $\nabla \gamma_{1} \wedge \nabla \gamma_{2} \in L^{1}$, not $L^{2}$ ), and also when one studies the problem of nonuniqueness of the representation.

Divergence-free vector fields appear often in fluid mechanics textbooks (these fields are called "solenoidal"), mostly as curls of other vector fields (recall that in three dimensions any curl has zero divergence). The idea of the representation $\vec{\jmath}=\nabla \gamma_{1} \wedge \nabla \gamma_{2}$ appears sometimes in a rather vague formulation. H. Lamb describes (in [5], Chapter VII, section 167) the structure of the solenoidal vector field given as the exterior product of the derivatives of the pressure and of the reciprocal of the density; he attributes the result to 


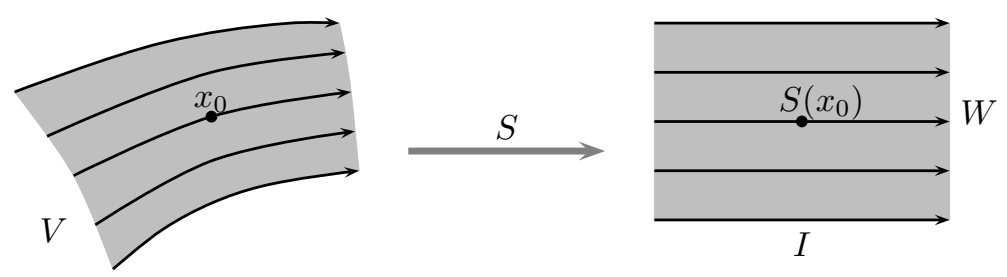

FIG. 1. Rectification of a smooth vector field

V. Bjerkenes, Vid.-Selsk. Skrifter, Kristiania, 1918. In [6], Section 3.12, M. Narasimhan leaves the proof of the existence of this representation as an exercise for the reader to carry out (Problem 1).

It should be noted that the representation $\vec{\jmath}=\nabla \gamma_{1} \wedge \nabla \gamma_{2}$ is related to the so-called Clebsch representation for velocity fields; see [5], 7] and [10].

In [12, the author states that any solenoidal vector field $\vec{\jmath}$ can be represented locally as $\nabla \gamma_{1} \wedge \nabla \gamma_{2}$. The proof presented is very intuitive and lacks mathematical rigour.

A purely geometric argument, based on differential forms, is given in [8]: the authors assert that any closed ( $n-1)$-form can be written (locally) as the exterior product of $n-1$ 1-forms. The authors make the following remark: "the representation of $\vec{\jmath}$ in terms of $n-1$ arbitrary functions is the most economical one" (the notation has been adapted). Unfortunately, the proof presented in $[8]$ is wrong.

The authors of [7] make the following assertion: "This representation of a divergencefree vector field was introduced by Euler."

The paper [4 gives a thorough discussion of three-dimensional solenoidal vector fields from the point of view of differential geometry.

5. The main representation result. We begin by presenting a result on arbitrary vector fields (having divergence not necessarily zero).

Theorem 5.1. Let $\vec{v}: \Omega \subset \mathbb{R}^{n} \rightarrow \mathbb{R}^{n}$ be a vector field of class $\mathcal{C}^{k}, k \geq 1$. Let $x_{0} \in \Omega$ be a point such that $\vec{v}\left(x_{0}\right) \neq \overrightarrow{0}$. There exists a neighbourhood $V$ of $x_{0}$ and scalar functions $\alpha \in \mathcal{C}^{k-1}(V)$ and $S_{2}, S_{3}, \ldots, S_{n} \in \mathcal{C}^{k}(V)$ such that

$$
\vec{v}(x)=\alpha(x) \nabla S_{2}(x) \wedge \nabla S_{3}(x) \wedge \cdots \wedge \nabla S_{n}, \forall x \in V .
$$

Proof. By a classical result of ordinary differential equations (rectification of a vector field; see [1, section 7, Theorem 1 and Corollary 10), there exists a diffeomorphism $S$ of class $\mathcal{C}^{k}$ between a neighbourhood $V$ of $x_{0}$ and an open subset of $\mathbb{R}^{n}$ of the form $I \times W$ (where $I$ is an open interval in $\mathbb{R}$, and $W$ is an open subset of $\mathbb{R}^{n-1}$; see Figure 1) with the property

$$
D S(x) \vec{v}(x)=\vec{e}_{1}, \quad \forall x \in V .
$$

In coordinate notation:

$$
\frac{\partial S_{i}}{\partial x_{j}} v_{j}=\nabla S_{i} \cdot \vec{v}=\delta_{1 i}, \quad i=1,2, \ldots, n
$$


We conclude that, for each $x \in V$, the $n-1$ vectors $\nabla S_{2}(x), \nabla S_{3}(x), \ldots, \nabla S_{n}(x)$ are orthogonal to $\vec{v}(x)$. As $S$ is a diffeomorphism, we know that the vectors $\nabla S_{i}(x)$, $i=1,2, \ldots, n$, are linearly independent. Hence, $\vec{v}(x)$ is collinear with the nonzero vector $\nabla S_{2}(x) \wedge \nabla S_{3}(x) \wedge \cdots \wedge \nabla S_{n}(x)$, so there is a scalar (which we denote by $\alpha(x)$ ) such that

$$
\vec{v}(x)=\alpha(x) \nabla S_{2}(x) \wedge \nabla S_{3}(x) \wedge \cdots \wedge \nabla S_{n}(x), \forall x \in V .
$$

The proof is almost completed; it remains only to prove that $\alpha \in \mathcal{C}^{k-1}(V)$. Consider the equality $\nabla S_{1} \cdot \vec{v}=1$ and replace $\vec{v}$ by (5.1) in order to obtain

$$
1=\nabla S_{1} \cdot\left[\alpha \nabla S_{2} \wedge \nabla S_{3} \wedge \cdots \wedge \nabla S_{n}\right]=\alpha \operatorname{det} D S .
$$

We deduce that

$$
\alpha=\frac{1}{\operatorname{det} D S},
$$

which provides the desired regularity for $\alpha$ and concludes the proof.

Lemma 5.2. Let $W=\left[a_{2}, b_{2}\right] \times\left[a_{3}, b_{3}\right] \times \cdots \times\left[a_{n}, b_{n}\right] \subset \mathbb{R}^{n-1}$ and let $c \in \mathcal{C}^{k}(W), k \geq 1$. Then, there exists an application $\Phi: W \rightarrow \mathbb{R}^{n-1}$ of class $\mathcal{C}^{k}$ such that $\operatorname{det} D \Phi=c$.

The above result says that it is possible to deform a domain $W$ obeying a prescribed area deformation $c$. It is easy to generalize the result for any convex domain $W$.

Proof of Lemma [5.2. We denote by $y$ points in $W$ and by $y^{\prime}$ points in $\left[a_{3}, b_{3}\right] \times \cdots \times$ $\left[a_{n}, b_{n}\right]$; thus, $y=\left(y_{2}, y^{\prime}\right) \in W$.

There are many possible ways of constructing $\Phi$; we choose here to deform $W$ in the $y_{2}$ direction only. That is, we define $\Phi_{i}(y)=y_{i}$ for $i \geq 3$ and then build $\Phi_{2}$ by integrating $c$ in $y_{2}$ :

$$
\Phi_{2}\left(y_{2}, y^{\prime}\right)=\int_{a_{2}}^{y_{2}} c\left(s, y^{\prime}\right) \mathrm{d} s .
$$

It is easy to compute the derivatives of $\Phi$ :

$$
\begin{aligned}
& \frac{\partial \Phi_{i}}{\partial y_{j}}=\delta_{i j} \quad \text { for } i \geq 3, j \geq 2, \\
& \frac{\partial \Phi_{2}}{\partial y_{2}}=c, \\
& \frac{\partial \Phi_{2}}{\partial y_{j}}\left(y_{2}, y^{\prime}\right)=\int_{a_{2}}^{y_{2}} \frac{\partial c}{\partial y_{j}}\left(s, y^{\prime}\right) \mathrm{d} s \quad \text { for } j \geq 3,
\end{aligned}
$$

and this concludes the proof.

Now we turn our attention to divergence-free vector fields:

Theorem 5.3. Let $\vec{\jmath}: \Omega \subset \mathbb{R}^{n} \rightarrow \mathbb{R}^{n}$ be a $\mathcal{C}^{k}$ vector field having zero divergence $(k \geq 2)$. Let $x_{0} \in \Omega$ be a point such that $\vec{\jmath}\left(x_{0}\right) \neq \overrightarrow{0}$. Then there exists a neighbourhood $V$ of $x_{0}$ and $n-1$ scalar functions $\gamma_{1}, \gamma_{2}, \ldots, \gamma_{n-1} \in \mathcal{C}^{k-1}(V)$ such that

$$
\vec{\jmath}(x)=\nabla \gamma_{1}(x) \wedge \nabla \gamma_{2}(x) \wedge \cdots \wedge \nabla \gamma_{n-1}(x), \forall x \in V .
$$

Proof. Consider the representation given in Theorem 5.1

$$
\vec{\jmath}(x)=\alpha(x) \nabla S_{2}(x) \wedge \nabla S_{3}(x) \wedge \cdots \wedge \nabla S_{n} .
$$


The idea of the proof is to show that, for divergence-free vector fields, it is possible to choose the functions $S_{2}, S_{3}, \ldots, S_{n}$ in such a way that the function $\alpha$ becomes equal to 1. One has:

$$
\begin{aligned}
\operatorname{div} \vec{\jmath} & =\operatorname{div}\left(\alpha \nabla S_{2} \wedge \nabla S_{3} \wedge \cdots \wedge \nabla S_{n}\right) \\
& =\nabla \alpha \cdot \nabla S_{2} \wedge \nabla S_{3} \wedge \cdots \wedge \nabla S_{n}+\alpha \operatorname{div}\left(\nabla S_{2} \wedge \nabla S_{3} \wedge \cdots \wedge \nabla S_{n}\right) .
\end{aligned}
$$

But the exterior product of any $n-1$ gradients has zero divergence; thus

$$
\operatorname{div} \vec{\jmath}=\nabla \alpha \cdot \nabla S_{2} \wedge \nabla S_{3} \wedge \cdots \wedge \nabla S_{n}=\frac{1}{\alpha} \nabla \alpha \cdot \vec{\jmath}
$$

( $\alpha$ is nonzero by construction). So, $\operatorname{div} \vec{\jmath}=0$ implies $\nabla \alpha \perp \vec{\jmath}$ in each point of $V$. This means that the function $\alpha$ is constant along trajectories of $\vec{\jmath}$. Transporting this information through the diffeomorphism $S$, we conclude that the function $\alpha \circ S^{-1} \in$ $\mathcal{C}^{k-1}(I \times W)$ is constant along $I$; it depends only on $y \in W$. We introduce the function $c \in \mathcal{C}^{k-1}(W)$ defined as $c(y)=\alpha\left(S^{-1}(\zeta, y)\right)$ for every $y \in W$ and for arbitrary $\zeta \in I$. Now apply Lemma 2 (one can choose a smaller $W$ which is the product of $n-1$ intervals) in order to obtain $\Phi: W \rightarrow \mathbb{R}^{n-1}$ of class $\mathcal{C}^{k-1}$ such that $\operatorname{det} D \Phi=c$. Denote by $\gamma_{1}, \gamma_{2}, \ldots, \gamma_{n-1}$ the components of $\Phi \circ S$ and compute

$$
\begin{aligned}
\nabla \gamma_{1} \wedge \nabla \gamma_{2} \wedge \cdots \wedge \nabla \gamma_{n-1} & =(\operatorname{det} D \Phi) \circ S \nabla S_{2} \wedge \nabla S_{3} \wedge \cdots \wedge \nabla S_{n} \\
& =\alpha \nabla S_{2} \wedge \nabla S_{3} \wedge \cdots \wedge \nabla S_{n}=\vec{\jmath} .
\end{aligned}
$$

The proof is complete.

In the above proof, one can see the nonunique character of the representation (5.2). Let us denote by $U=\Phi(W)$ the domain where the function $\gamma=\left(\gamma_{1}, \gamma_{2}, \ldots, \gamma_{n-1}\right)$ takes values. It is an open set (as the vectors $\nabla \gamma_{1}\left(x_{0}\right), \nabla \gamma_{2}\left(x_{0}\right), \ldots, \nabla \gamma_{n-1}\left(x_{0}\right)$ are linearly independent; one can choose a smaller $V$ if necessary). If $\Psi: U \rightarrow \mathbb{R}^{n-1}$ is a $\mathcal{C}^{k}$ application such that $\operatorname{det} D \Psi$ is constantly equal to 1 (a volume-preserving map), then the components of $\Psi \circ \Phi \circ S$ still enjoy the same property (the exterior product of their gradients equals $\vec{\jmath}$ ). That is, we can characterize the nonuniqueness of $\gamma$ as follows: one may compose $\gamma$ with an arbitrary volume-preserving application in $\mathbb{R}^{n-1}$.

6. Future work and challenges. The main limitation of the result stated in Theorem 5.3 is the nondegeneracy hypothesis $\vec{\jmath}\left(x_{0}\right) \neq \overrightarrow{0}$. Unfortunately, it seems that this hypothesis cannot be eliminated; see the counterexample presented in [7]. The necessity of this hypothesis discourages also any attempt of proving a global representation result.

Another possible generalization of Theorem 5.3 is to lower the regularity of the functions involved. For instance, it is not clear what kind of representation one should expect for vector fields $\vec{\jmath}$ whose components are in $L^{1}$ or in $L^{2}$. Again, the hypothesis $\vec{\jmath}\left(x_{0}\right) \neq \overrightarrow{0}$ looks discouraging since such a vector field is defined almost everywhere. A first step could be to state and prove some estimates on the potentials $\gamma_{i}$, in an appropriate norm.

The author thinks that the following conjecture is true, under appropriate hypotheses. The conjecture is stated here only for three dimensions to simplify the formulae, but it should hold true in arbitrary dimensions. Let $\vec{\jmath}, \vec{\sigma}$ and $\vec{\tau}$ be three divergence-free vector fields in $\mathbb{R}^{3}$. Then there are three scalar potentials $\gamma_{1}, \gamma_{2}$ and $\gamma_{3}$ such that, locally, $\vec{\jmath}=\nabla \gamma_{2} \wedge \nabla \gamma_{3}, \vec{\sigma}=\nabla \gamma_{3} \wedge \nabla \gamma_{1}$ and $\vec{\tau}=\nabla \gamma_{1} \wedge \nabla \gamma_{2}$. 
Finally, the numerical treatment of partial differential equations is another challenge for the future. In elliptic problems, a state equation of the form $\operatorname{div}(a \nabla u)=0$ can be replaced by $a \nabla u=R \varphi$ if $n=2$, or by $a \nabla u=\nabla \gamma_{1} \wedge \nabla \gamma_{2}$ if $n=3$. New unknown functions are thus introduced, and some of the boundary conditions should be reformulated in terms of the new unknowns. See also [2].

Acknowledgements. This work was supported by Fundação para a Ciência e a Tecnologia, Financiamento Base 2008 - ISFL/1/209.

The author had a lot of useful discussions on this topic with many people: Armando Machado, Luc Tartar, Hugo Beirão da Veiga, Bernard Dacorogna, Vivette Girault, Filipe Oliveira, José Bellido, and the reviewer of the paper. Many thanks for their comments, suggestions and corrections.

\section{REFERENCES}

[1] V.I. Arnol'd, Ordinary differential equations, Springer-Verlag, 1992. MR1162307 (93b:34001)

[2] G. Auchmuty, J.C. Alexander, Finite-Energy Solutions of Mixed 3D div-curl systems, Quart. Appl. Math. 64 (2006), 335-357. MR2243867 (2007b:35043)

[3] W.H. Fleming, Functions of several variables, Addison-Wesley, 1965. MR0174675 (30:4875)

[4] R. Kotiuga, Clebsch potentials and the visualization of three-dimensional solenoidal vector fields, IEEE Transactions on Magnetics 27 (1991), no. 5, 3986-3989.

[5] H. Lamb, Hydrodynamics, Cambridge University Press, 1974. MR.1317348 (96f:76001)

[6] M. Narasimhan, Principles of continuum mechanics, Wiley, 1993. MR1198890 (94b:73001)

[7] F. Henyey, R. Graham, Clebsch representation near points where the vorticity vanishes, Physics of Fluids 12 (2000), no. 4, 744-746. MR1745900 (2000k:76033)

[8] K. Swaminathan, S. D. Nigam, R. Usha, Divergence-free vector fields, Jour. Math. Phy. Sci. 14 (1980), no. 5, 523-527. MR644272 (83h:53028)

[9] P.A. Raviart, V. Girault, Finite element approximation of the Navier-Stokes equations, Lecture Notes in Mathematics, no. 749, Springer-Verlag, 1979. MR.548867 (83b:65122)

[10] R.L. Seliger, G.B. Whitham, Variational Principles in Continuum Mechanics, Proc. Roy. Soc. A 305 (1968), 1-25.

[11] G. Strang, Calculus, Wellesley-Cambridge, 1991.

[12] C.-S. Yih, Stream functions in three-dimensional flows, La Houille Blanche 3 (1957), 445-450. MR0090287 (19:794d) 